\title{
A Low Power Low Noise Chopper-Stabilized Tow-stage Operational Amplifier for Portable Bio-potential Acquisition Systems Using 90 nm Technology
}

\author{
Fateh Moulahcene ${ }^{1}$, Nour-Eddine Bouguechal ${ }^{1}$ and Youcef Belhadji ${ }^{2}$ \\ ${ }^{1}$ Advanced Electronics Laboratory (LEA), University of Batna, Avenue Chahid \\ Boukhlouf, 05000, Batna,Algeria \\ Micro30.sysfateh@gmail.com \\ ${ }^{2}$ Unit of research of materials and renevable energies, faculty of science, \\ Abou bekr Belkaid University, \\ PB 119 Tlemcen 13000, Algeria
}

\begin{abstract}
This paper presents a high performance chopper-Stabilized Two-stage operational amplifier for biomedical applications. This Two-stage is designed for low noise, low power, high PSRR and high CMRR. The Miller compensation technique $(C c)$ is used with a nulling active resistance $(R z)$ implemented using Transmission gate (TG) transistors for stable operation in feedback mode. Chopper stabilization technique has been widely used in amplifiers for flicker (1/f) noise and offsets reduction purposes using the principles of modulation and demodulation. Thus, the functionality and performance of modulation and demodulation circuits determines the realization and attainment of chopper stabilization. The operational amplifier was manufactured in a SPECTRE using GPDK 90nm CMOS technology with threshold voltages of a $0.17 \mathrm{~V}$ and $-0.14 \mathrm{~V}$ achieve a low power 2.6uW, $13.5 \mathrm{nV} / \sqrt{\mathrm{Hz}}$ at $10 \mathrm{~Hz}$ high CMRR up to $130 \mathrm{~dB}$ and PSRR up to $70 \mathrm{~dB}$ at $1 \mathrm{~V}$ power supply.
\end{abstract}

Keywords: Two-stage amplifier, Miller compensation, Transmission gate, CMRR (Common mode Rejection Ratio), Modulation, Demodulation, Chopper stabilization

\section{Introduction}

The trend in the use of low-voltage power supplies for CMOS integrated circuits is by the reliability issue of small size MOSFET transistors and the increasing use of low-weight long life battery-operated portable electronic systems $[1,2]$.

With the rapid development of microelectronics in the recent past years, more and more applications require an ultra-low amplitude signal measurement module, such as implantable devices in biomedical application to monitor several Neuro-muscular activities [3-6].

Monitoring biomedical signals of the human body is a very interesting topic since it can be used to know vital health information of the body from the acquired data. These data capacitates medical practitioners to diagnose diseases [7, 8].

Biomedical signals, such as electrocardiogram (ECG), Electromyogram (EMG), Electroencephalogm (EEG), are characterized by their low voltage-levels and low frequency, Table 1 shows the characteristics of these signals [8 -12]. 
Table 1. Most Commonly used Biomedical Signals

\begin{tabular}{|c|c|c|}
\hline Signal & Frequency & Amplitude \\
\hline ECG & $0.05-250 \mathrm{~Hz}$ & $5 \mathrm{uV}-8 \mathrm{mV}$ \\
\hline EEG & $0.5-200 \mathrm{~Hz}$ & $2 \mathrm{uV}-200 \mathrm{uV}$ \\
\hline EMG & $0.01-10 \mathrm{KHz}$ & $50 \mathrm{uV}-10 \mathrm{mV}$ \\
\hline
\end{tabular}

A low noise and low power consumption amplifier is one of the key circuits for detecting the small level signals in the biomedical applications. However, in CMOS technology the $1 / \mathrm{f}$ noise becomes a serious problem which limits the minimum detectable signal in amplifier at low frequency $[13,14]$. The chopping technique is widely used in amplifier for low noise. It is a modulation and demodulation technique that can be employed to reduce the effect of operational amplifier imperfections including noise and input referred dc offset voltage [15].

The Famous architecture used in a biomedical monitoring system, are an operational amplifier, must exhibit very low-referred noise, low power and high Common Mode Rejection Ratio (CMRR).

In this paper, a low voltage, low noise and high CMRR operational amplifier for portable monitoring system is proposed. The operational amplifier is able to work under 1-V supply, has high CMRR and low noise.

\section{ECG Data Acquisition System}

ECG data acquisition begins with the use of electrodes that attached to body skin. Three electrodes are used as sensors to detect the heart signals from a human body. Two electrodes are placed each on the left and right wrist while the third electrode is placed on the ankle of the leg as ground [16].

The ECG signal that acquired is in the range of $5 \mathrm{uV}$ to $8 \mathrm{mV}$. Due to the weak voltage level, the signal is fed into an amplifier circuit to be amplified to a desirable voltage level. Output from the amplifier is then fed into a bandpass filter circuit and a High Q notch filter. The purpose of this filter is to filter out the very low and high frequency noise components of the signals and the $60-\mathrm{Hz}$ power line interference. The desirable analog output from the filter is then sent to $\mathrm{S} / \mathrm{H}$ and $\mathrm{ADC}$ to become a digital signal. After that, these digital data will processed in PCs or microprocessors. Figure.1 shows the proposed ECG data acquisition system [7, 16-19].

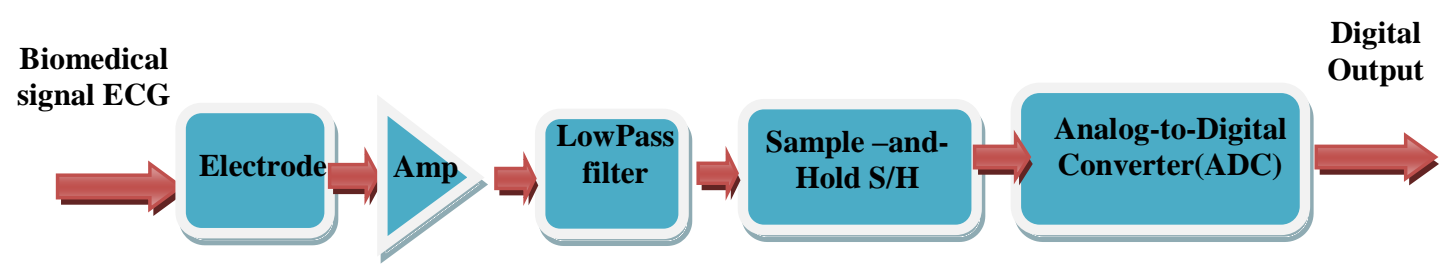

Figure 1. The Proposed ECG Data Acquisition

We have collected data from MIT-BIH Arrhythmia Database [20-22], for training and testing of proposed ECG amplifier Design. See Figure 2 of the input (VIN) ECG signal.

\section{ECG Amplifier Design}

A normal ECG signal falls in the range of $5 \mathrm{uV}-8 \mathrm{mV}$. The amplifier is required to increase this weak signal into an acceptable level. The main operational amplifier for 
biomedical applications is illustrated in Figure 3. This Two-stage architecture with miller compensation capacitor $(\mathrm{Cc})$ and nulling active resistor $(\mathrm{Rz})$, is used for the design of the operational amplifier with the first stage being a differential input pair and the second, a gain stage.

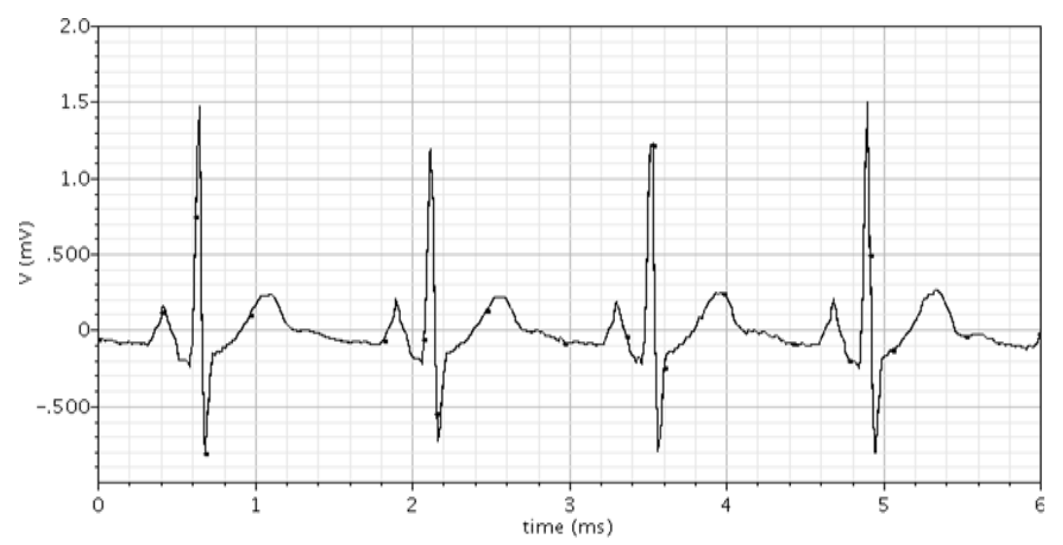

Figure 2. ECG Signal from MIT-BIH Arrhythmia Database (Vin $(\max )=1.5 \mathrm{mV})$

This Two-stage architecture with miller compensation capacitor (Cc) and nulling active resistor $(\mathrm{Rz})$, is used for the design of the operational amplifier with the first stage being a differential input pair and the second, a gain stage. Because the Biomedical signal is so weak, the noise will affect the real biomedical signal. Due to the low frequency of biomedical signal the flicker noise dominates, it has strong dependence on the width and length product of a CMOS transistor. The flicker noise is modeled in Eq (1), the spectral density of flicker noise is inversely proportional to the transistor area, WL. In other words, $1 / \mathrm{f}$ noise can have a lesser effect on larger devices. However the input pair (M1 and M2) of the input stage has been designed using large PMOS transistors. A P-channel current source (M5) and an n-channel current mirror load (M3 and M4) are used in the input stage.

$$
V_{n}^{2}=\frac{K}{C_{o x} W L} \cdot \frac{1}{f}
$$

The second stage of the operational amplifier includes an n-channel common-source amplifier (M6) with a p-channel current source load (M7).

The high output resistances of these two transistors (M7, M6) equate to a relatively large gain for this stage and an overall moderate gain for the complete amplifier. Because the operational amplifier inputs are connected to the gates of MOS transistors, the input resistance is essentially infinite. The sizes of the transistors were designed for a bias current of $0.5 \mu \mathrm{A}$ to provide for sufficient output voltage swing, output-offset voltage, slew rate, and gain-bandwidth product.

\subsection{Current Mirrors}

Current mirrors are used extensively in MOS analog circuits both as biasing elements and as active loads to obtain high AC voltage gain [23, 24]. Enhancement mode transistors remain in saturation when the gate is tied to the drain, as the drain-to source voltage (VDS) is greater than the gate-to-source voltage $(V G S)$ due to the threshold voltage (Vth) drop, i.e., 


$$
V_{D S}=V_{G S}-V_{T h}
$$

Based on Eq (2), constant current sources are obtained through current mirrors designed by passing a reference current through a diode-connected (gate tied to drain) transistor. We choose a simple current mirror because low voltage (1-V), Figures 4(a) and (b) show the PMOS and NMOS current mirrors design. A PMOS mirror serve as a current source while the NMOS acts as a current sink.

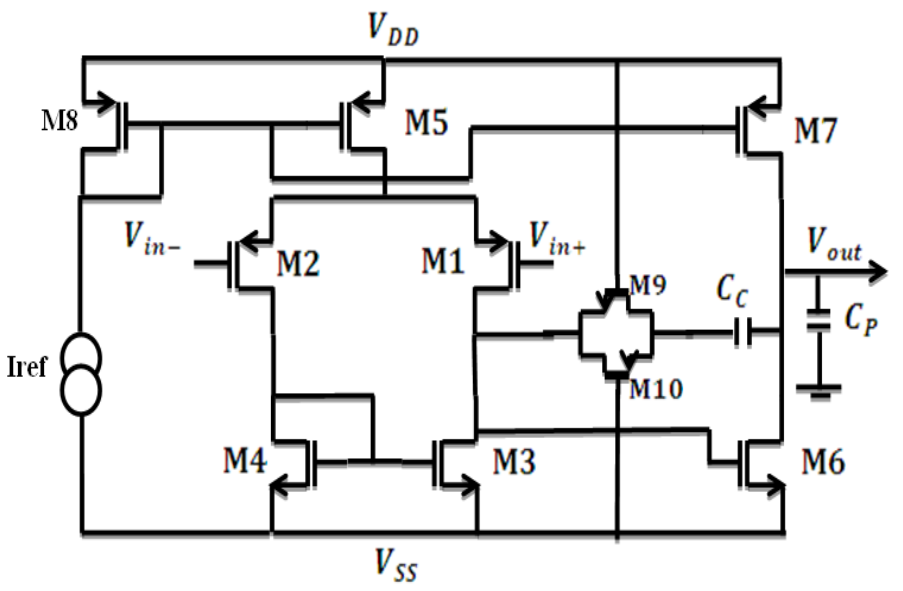

Figure 3. Schematic of Two-stage Operational Amplifier

The voltage developed across the diode-connected transistor is applied to the gate and the source of the second transistor, which provides a constant output current. Since both the transistors have the same gate to source voltage, the currents when both transistors are in the saturation region of operation, are governed by the following Eq (3) and Eq(4) assuming matched transistors. The current ratio Iout/Iref is determined by the aspect ratios of the transistors. The reference current that was used in the design is $0.5 \mu \mathrm{A}$. The desired output current is $1 \mu \mathrm{A}$.

For the PMOS current mirror, we can write,

$$
\frac{I_{\text {OUT }}}{I_{\text {REF }}}=\frac{W_{7}}{L_{7}} / \frac{W_{8}}{L_{8}}
$$

For the NMOS current mirror, we can write,

$$
\frac{I_{\text {OUT }}}{I_{\text {REF }}}=\frac{W_{4}}{L_{4}} / \frac{W_{3}}{L_{3}}
$$



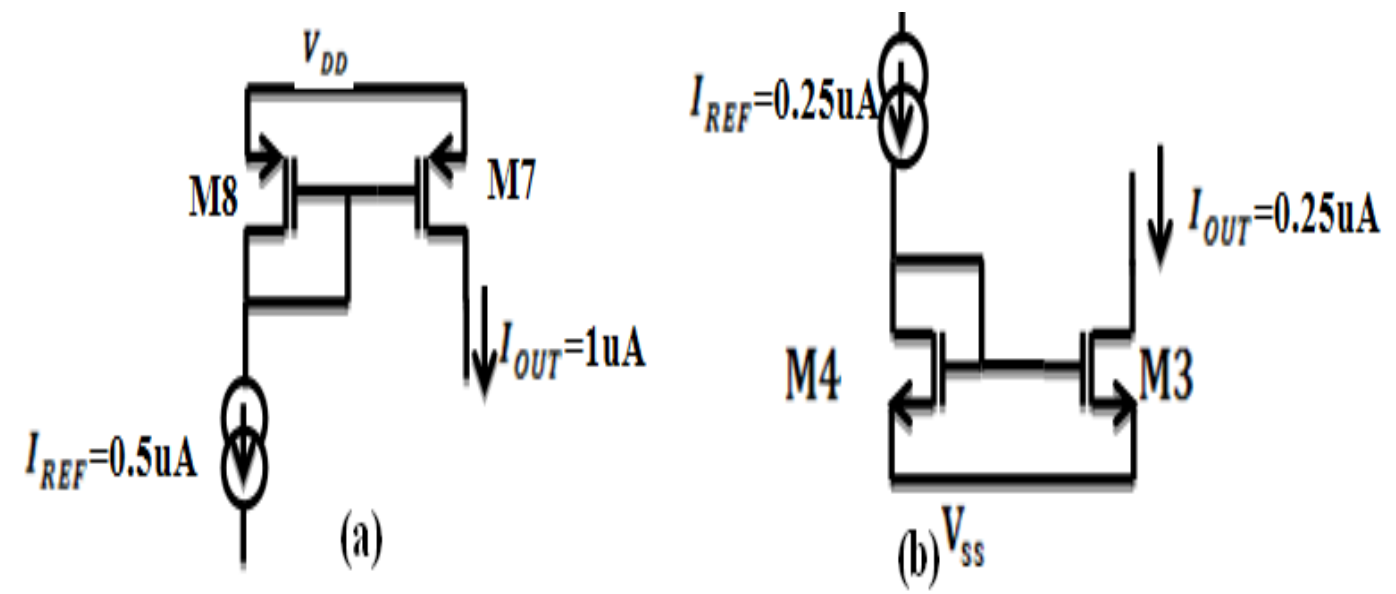

Figure 4. (a) PMOS Current Mirror, (b) NMOS Current Mirror

\subsection{Active Resistors}

There are two active resistors used in the design. Firstly, the reference current that is applied to the current mirror is obtained by means of an active resistor. The resistor here is obtained by simply connecting the gate of an NMOS (M11) to its drain as shown in Figure 5. This connection forces the MOSFET to operate in saturation.

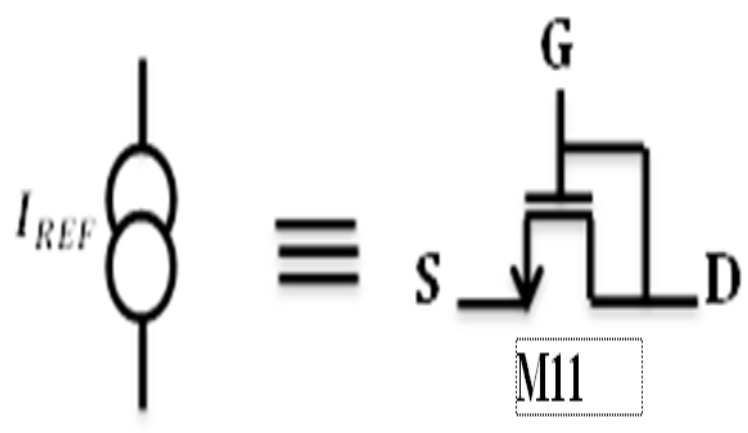

Figure 5. Active Resistor

The second active resistor a transmission gate (TG) shown in Figure 7 has been used to realize the nulling active resistance $(\mathrm{Rz})$ to reduce the effects of the right hand plane zero in the transfer function. The gate of these transistors M9, M10 is biased at VDD, VSS respectively. Its small signal output resistance is obtained from $\mathrm{Eq}(5)$ and $\mathrm{Eq}(6)\left(\mathrm{V}_{\mathrm{ds}}\right.$ very small).

The dynamic range limitations associated with single channel MOS switches can be avoided with CMOS switch shown in figure 6. Using the CMOS technology, a switch usually constructed by connecting P-channel and $\mathrm{N}$-channel enhancement mode in parallel as illustrated. 


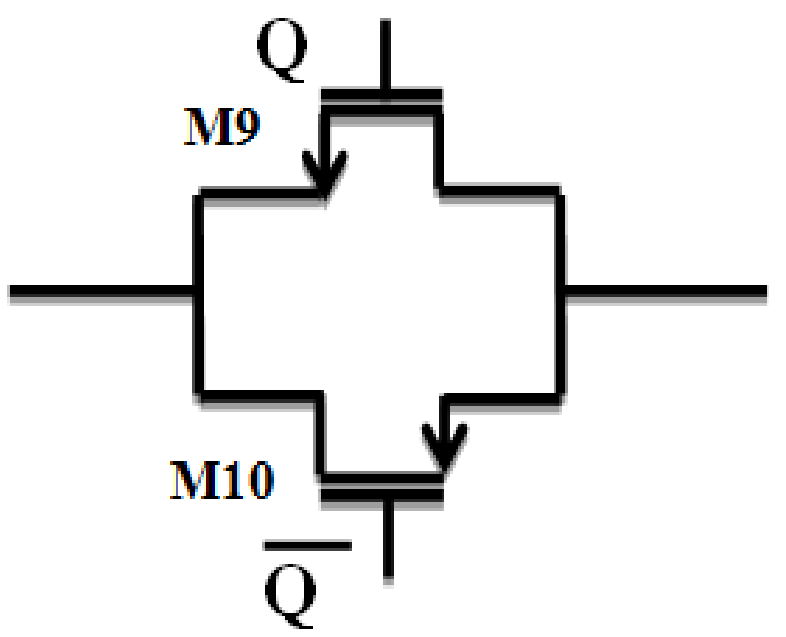

Figure 6. Transmission Gate (TG)

For this configuration, When $\mathrm{Q}$ is low; both transistors are off, creating an effective open circuit. When $\mathrm{Q}$ is high both transistors are on, giving a low impedance state. The bulk potentials of the p-channel and n-channel devices are taken at the highest and lowest potentials respectively. The primary advantage of the CMOS switch over the single channel MOS switch is that the dynamic analog-single range in the ON state is greatly increased [24].

$$
\begin{aligned}
R_{O N} & =\frac{1}{U_{n} C_{O X} \frac{W}{L}\left(V_{G S}-V_{T h n}\right)} \\
R_{O P} & =\frac{1}{U_{P} C_{O X} \frac{W}{L}\left(V_{S G}-\left|V_{T h P}\right|\right)}
\end{aligned}
$$

\section{Chopper Stabilization Structure}

CMOS technology suffers from a specific type of noise namely flicker noise is concentrated in low frequency bands. It arises from the channel of the MOS transistor, due to the imperfect silicon crystal structure at the interface between the gate oxide and the silicon substrate [13]. Amongst the technique used to reduce the effect of such noise type is chopper stabilization technique, when compared to other published techniques, chopper stabilization efficiently reduces the flicker noise in the design of an analog circuit [14]. In Figure 7, we show the theory of the chopper stabilization structure. 


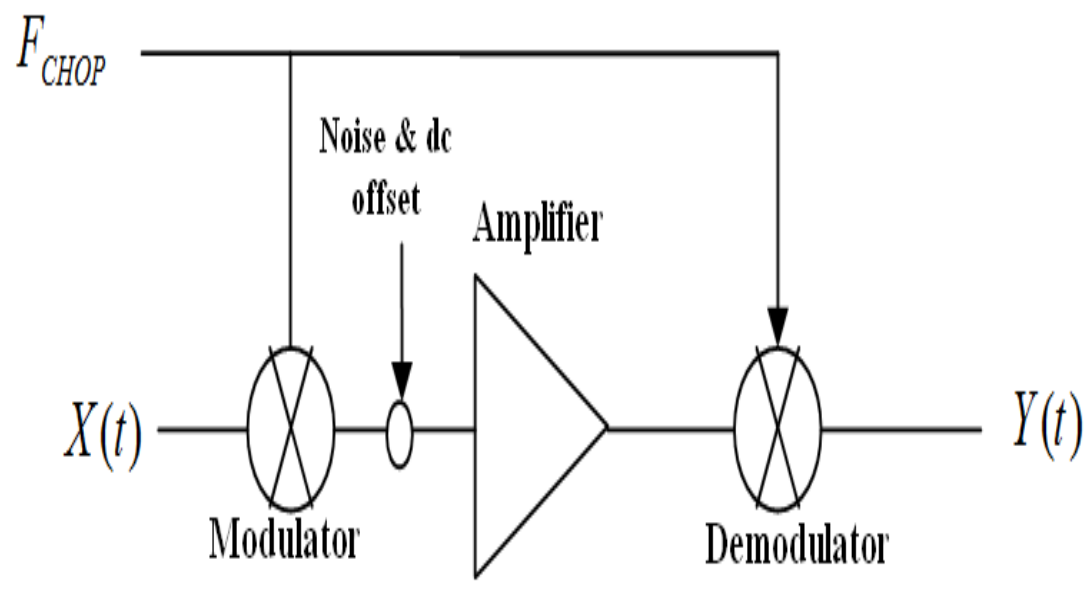

Figure 7. Chopper Stabilisation Structure

In Figure 7, the input signal is firstly modulated by the chopping signal with the chopping frequency Fchop and shifted to odd harmonic of the chopping frequency. And after amplification it is demodulated to the even harmonics. However, the noise and DC offset before the amplifier is just modulated once and shifted to the odd harmonics of chopping frequency. It leaves the thermal noise at the base-band and reduces much of flicker noise. Figure 8 shows the signal and noise wave at the node of chopper stabilization structure. Through the chopper stabilization technique, we can reduce the low frequency noise and DC offset effectively [25].

\subsection{Modulation and Demodulation Circuit}

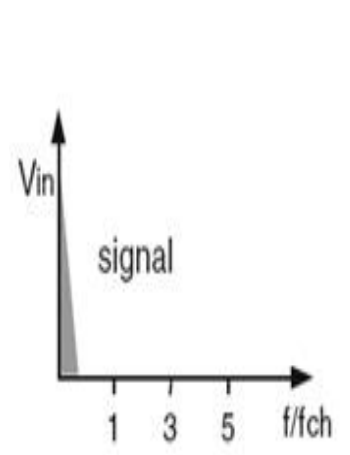

(a)

\section{offset\& noise}

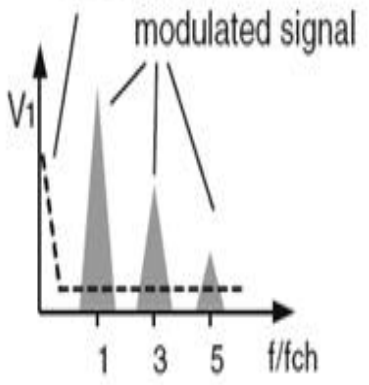

(b)

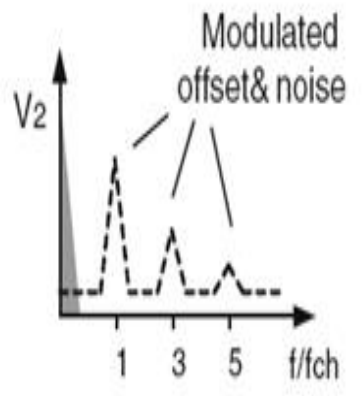

(c)

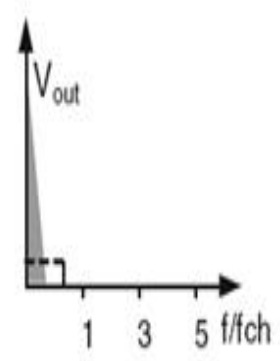

(d)

Figure 8. Chopping Principal in Frequency Domain

For the switches of modulation and demodulation circuit, the structure of differential NMOS switch which is shown in figure 9 is used in this design [26, 27] .

The modulator inverts the signal when $\mathrm{Q}$ is low and does not invert the signal when $\mathrm{Q}$ is high. The demodulator is identical. However, the modulator and demodulator can be independently controlled which makes it possible to adjust for phase delay problems 
The modulator circuit, which is placed before the chopper amplifier, is critical to range of nonlinearities and noise. Hence for low noise Two-stage operational amplifier applications, critical attention should be given to the design of the modulator part for better noise and offset minimization.

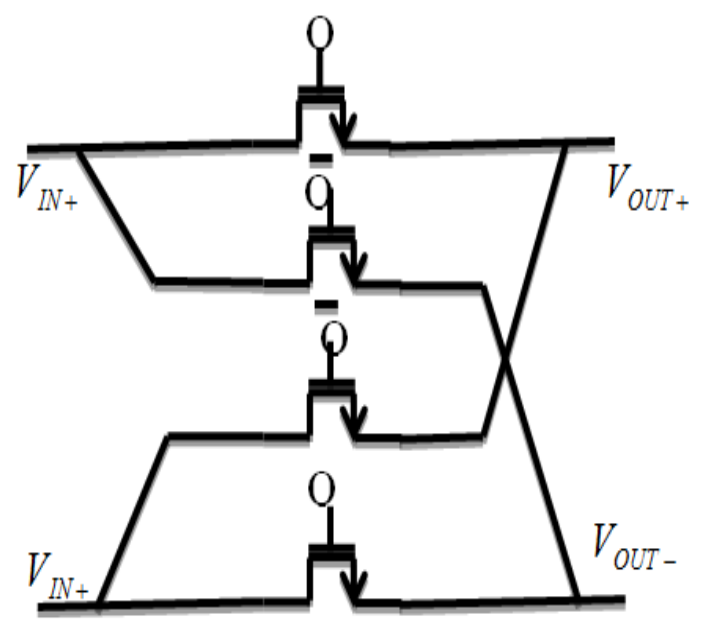

Figure 9. Differential NMOS Switch

Clock feed-through and charge injection are the main factors resulting in nonlinearity in MOS switches .figure 10 shows differential NMOS chopper circuit with half size dummy switchs incorporation [27-29].

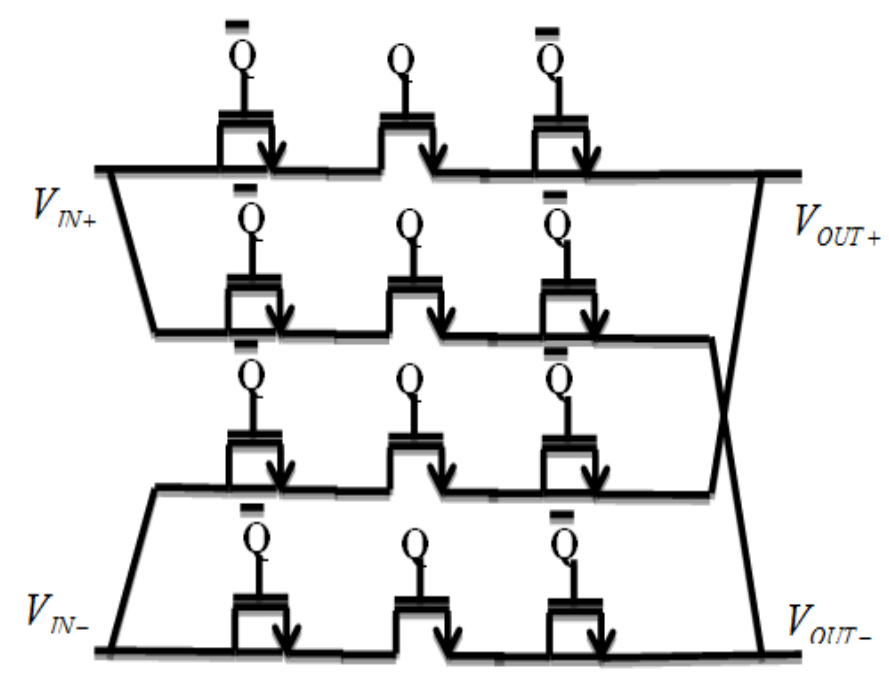

Figure 10. Differential Chopper Circuit with Dummy Switches

\section{Simulation and Discussion}

The proposed two-stage operational amplifier consists of a differential difference amplifier and a chopper circuit. The following subsections explain the simulation of each module. 


\subsection{Device Parameter}

Table 2 gives the device parameter for a Two-stage operational amplifier.

Table 2. Device Parameter for Two-stage Amplifier

\begin{tabular}{|c|c|c|c|}
\hline Device & Type & W(um) & L(um) \\
\hline M1,M2 & P & 10 & 0.378 \\
\hline M3,M4 & N & 18 & 0.556 \\
\hline M5,M8 & P & 35 & 0.460 \\
\hline M7 & P & 80 & 0.530 \\
\hline M6 & N & 15 & 0.100 \\
\hline M9 & N & 1 & 0.100 \\
\hline M10 & P & 10 & 0.100 \\
\hline M11 & N & 3 & 0.800 \\
\hline
\end{tabular}

\subsection{Frequency Response, Compensation}

Operational amplifier architectures are generally two types: single-stage externally compensated or two stage internally compensated amplifiers, both of which can have a dominant two pole type frequency response [30]. Ignoring higher order poles, the small signal behavior of the two stage amplifier can be modeled by the general two poles, one zero small signal equivalent circuit shown in Figure 11. Referring back to the model of the input stage Figure 3 (Without $(\mathrm{Cc})$ and $(\mathrm{Rz})$ ), We have two poles these are Eq (7) and Eq (8)

$$
\begin{gathered}
P_{1}=\frac{1}{C_{1} \frac{1}{g_{m 3}}} \\
P_{2}=\frac{1}{C_{2}\left(r_{02} r_{04}\right)}
\end{gathered}
$$

The compensation of the two stage CMOS amplifier can be carried out using a pole splitting capacitor. The goal of the compensation task is to achieve a phase margin greater than 45 degree. The circuit can be approximately represented by the small signal equivalent circuit of Figure 11 (without Rz), if the non-dominant poles which may exist on the circuit are neglected.

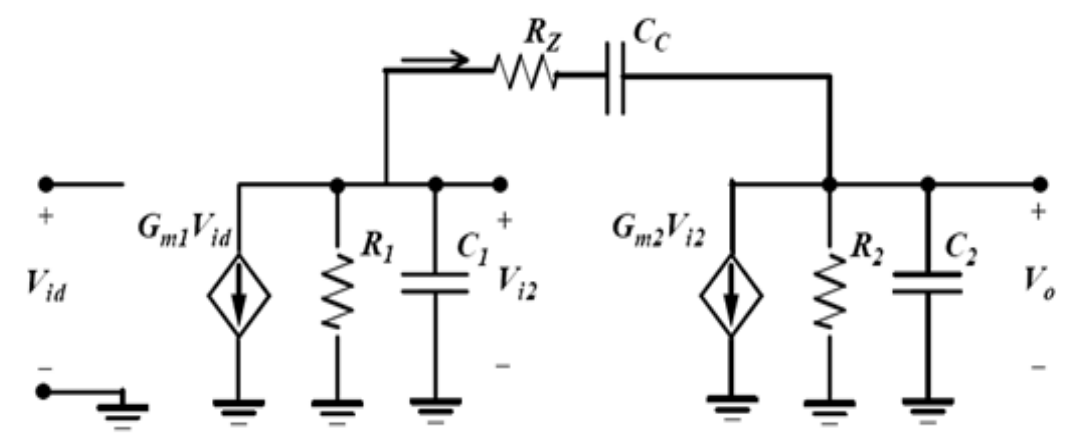

Figure 11. Simplified Small Signal Model of the Basic Two Stage Op-amp Added with The Nulling Resistor 
The circuit displays two poles and a half right plane zero, which under an assumption that the poles are widely separated can be shown to be approximately located at (Eq (9), Eq (10) and $\mathrm{Eq}(11)$ ).

$$
\begin{aligned}
P_{1} & =\frac{-1}{\left(1+g_{m 2} R_{2}\right) C_{C} R_{1}} \\
P_{2} & =\frac{-g_{m 2} C_{C}}{C_{2} C_{1}+C_{C} C_{1}+C_{2} C_{C}} \\
Z & =\frac{g_{m 2}}{C_{C}}
\end{aligned}
$$

Note that the pole due to the capacitive loading of the first stage by the second, $p 1$, has been pushed down to a very low frequency by the miller effect in the second stage, while the pole due to the capacitance at the output node of the second stage $p 2$, has been pushed to a very high frequency due to the shunt feedback. For this reason, the compensation technique is called pole splitting.

Physically, the zero arises because the compensation capacitor provides a path for the signal to propagate directly through the circuit to the output at high frequencies. Since there is no inversion in that signal path as there is in the inverting path dominant at low frequencies, stability degraded.

An even simpler approach is to insert an active nulling resistor $(\mathrm{Rz})$ in series with the compensation capacitor as shown in Figure 4 and Figure 8. If $R z$ is assumed to be less than $R 1$ or $R 2$ and the poles are widely spaced, then the poles are: $(\mathrm{Eq}(12), \mathrm{Eq}(13), \mathrm{Eq}(14)$ and $\mathrm{Eq}(15))$.

$$
\begin{aligned}
& P_{1}=\frac{-1}{\left(1+g_{m 2} R_{2}\right) C_{C} R_{1}} \cong \frac{-1}{g_{m 2} C_{C} R_{1} R_{2}} \\
& P_{2}=\frac{-g_{m 2} C_{C}}{C_{1} C_{2}+C_{C} C_{2}+C_{1} C_{C}} \cong \frac{-g_{m 2}}{C_{2}} \\
& P_{3}=\frac{-1}{R_{Z} C_{1}} \\
& Z=\frac{-1}{C_{C}\left(\frac{1}{g_{m 2}-R_{Z}}\right)}
\end{aligned}
$$

The resistor $\mathrm{RZ}=(\mathrm{Ron} \| \mathrm{Rop})$ allows independent control over the placement of the zero. The zero vanishes when RZ is made equal to $1 / \mathrm{gm} 2$. In fact, the resistor can be further increased to move the zero into the left half-plane and place it on top of $\mathrm{p} 2$ to improve the amplifier phase margin. RZ can be realized by a CMOS switch transistor in the triode region.

Using CMOS switch is to increase dynamic range. Where the resistance of CMOS switch is plotted as a function of the input voltage figure 12, in this figure, the p-channel M10 and nchannel M9 devices are sized in such a way that they have an equivalent resistance to identical terminal conditions. 
The peak behavior at midrange (near VDD/2), is due the parallel combination of the two devices M9 and M10. In the right n-channel M9 dominating when E is low, and in the left pchannel M10 dominating when $\mathrm{E}$ is high (near VDD).

Figure 13 shows a typical variation of the gain and phase versus frequency, with $\mathrm{Cc}=0.6$ $\mathrm{pF}, \mathrm{Cp}=1 \mathrm{pF}$.

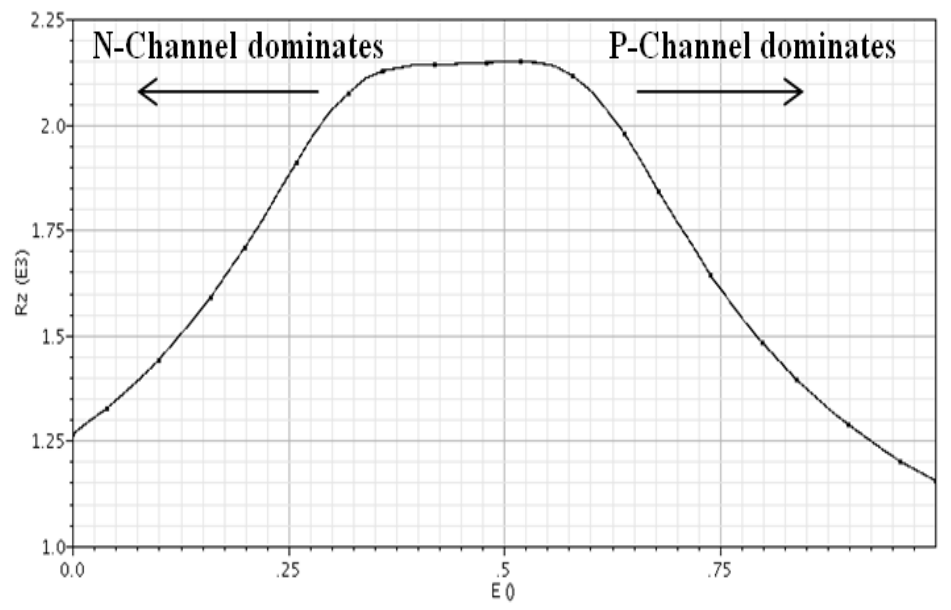

Figure 12. Rz of Figure 6, as a Function of E

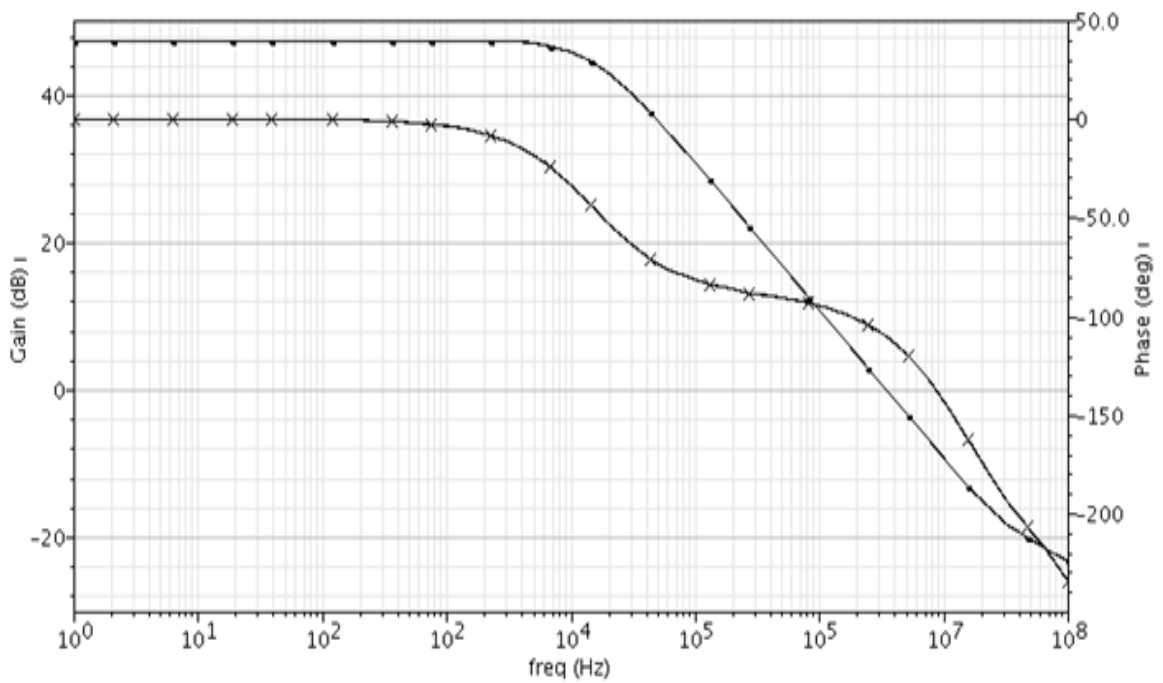

Figure 13. Shows a Typical Variation of the Gain and Phase versus Frequency

\subsection{Common Mode Rejection Ratio CMRR}

Due to the large amount of $60-\mathrm{Hz}$ human in bio-potential measurements, common-mode rejection is as important as low-noise operation. CMRR is defined as the ratio of the voltage gain for a differential-mode input signal to the voltage gain for a common-mode input signal, and can be stated as [22]. Therefore, measuring the dc CMRR involves determining the change in the offset voltage due to any change in the applied common-mode voltage. But, power supply voltage variation may affect the output as well as the offset voltage. In order to 
ensure that the measured change in the offset voltage is only due to a change in commonmode input voltage, the output voltage of the Two-stage operational amplifier is maintained at a fixed voltage for the complete test sequence.

We simulate the complete circuit in single power supply for CMRR. The results of CMRR are up to $130 \mathrm{~dB}$ as shown in Figure 14.

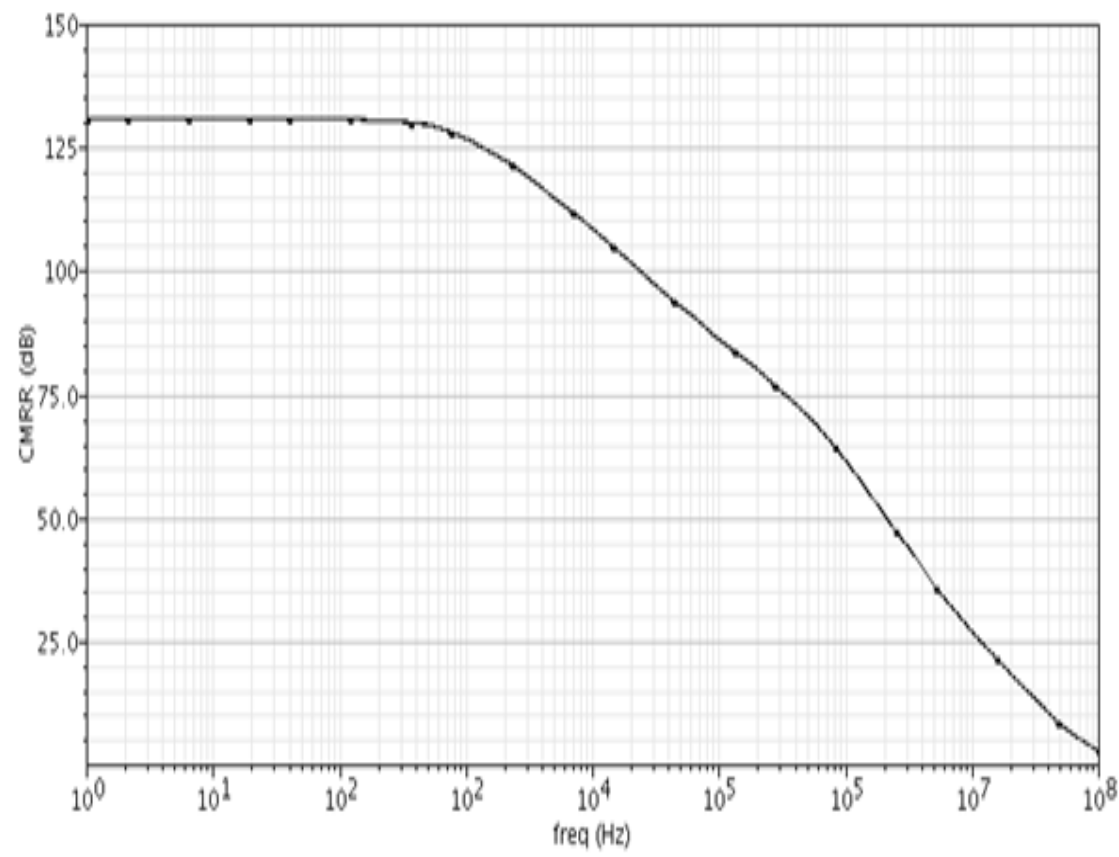

Figure 14. Shows a Variation of the CMRR versus Frequency

\subsection{Noise}

The input stage dominates the noise of the op amp. PMOS devices offer lower flicker noise which is advantageous in our op amp design. Also, the noise component of the load devices is scaled by the ratio of their transconductance to that of the input pair devices. So, the transconductance of the input differential pair needs to be larger than that of the load pair in order to ensure low input-referred noise. There exists a trade-off between noise and the output swing of the stage. Generally, the overdrive voltage of the current loads is minimized to realize a wide output swing, but for a fixed current bias this increases the transconductance (due to increased $\mathrm{W}$ ) and thereby results in a larger input-referred noise.

The simulation results of the input referred noise spectrum is depicted in Figure 15, flicker noise below $100 \mathrm{~Hz}$ is clearly visible, it achieve input referred noise density of $138 \mathrm{nV} \sqrt{\mathrm{Hz}}$ at $10 \mathrm{~Hz}$. 


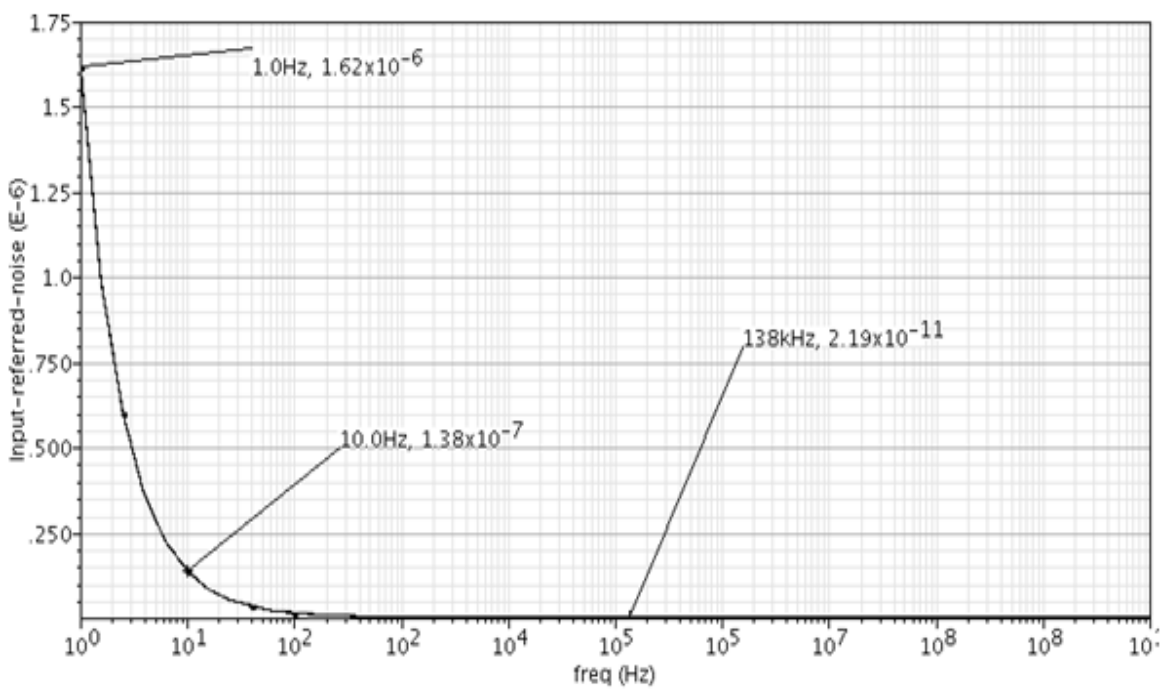

Figure 15. Simulated Input-refred Noise Spectrum

\subsection{Chopper Circuit}

The simulation result of the modulator and demodulator circuits in Cadence Virtuoso Spectre circuit simulator showed that the differential NMOS chopper circuit implantation exhibit large charge injections and spikes Whereas, the dummy switch incorporated chopper circuit results in smooth modulated signal output as shown in Figure 16, in Figure 16 is the output modulator signal with dummy switches and Figure 17 is the output of our operational amplifier without low pass filter. The chopper clock is chosen to be a $10 \mathrm{KHz}$ square-wave and the input is $0.5 \mathrm{KHz}$ sinusoidal signal.

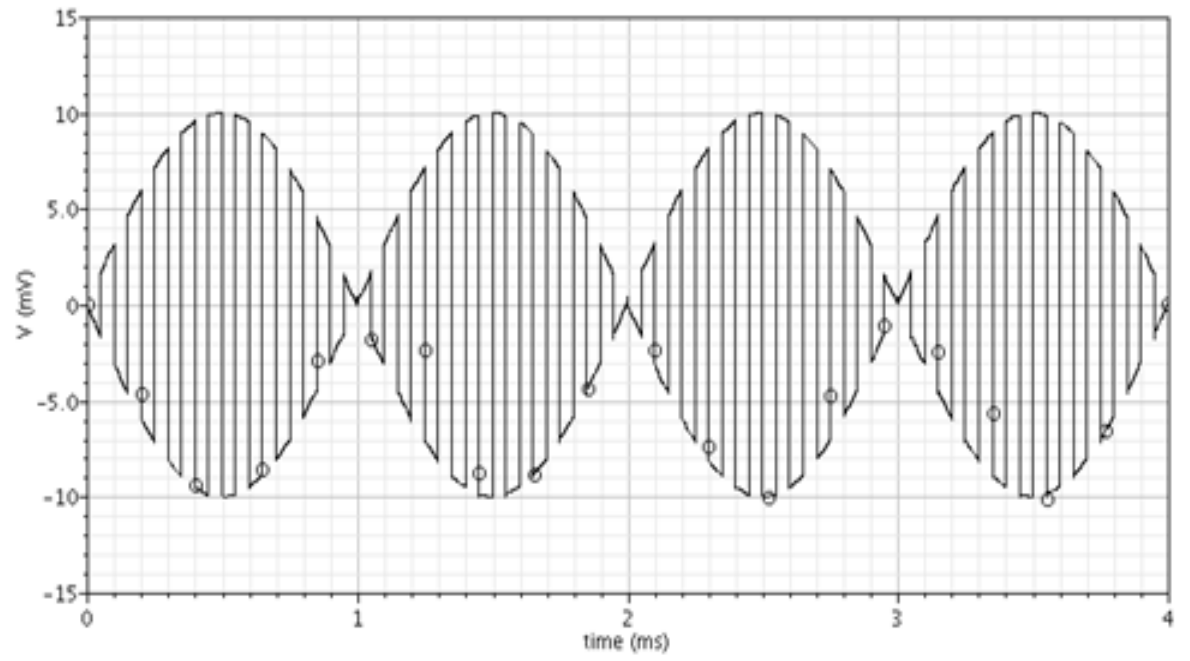

Figure 16. Output Modulated Signal 


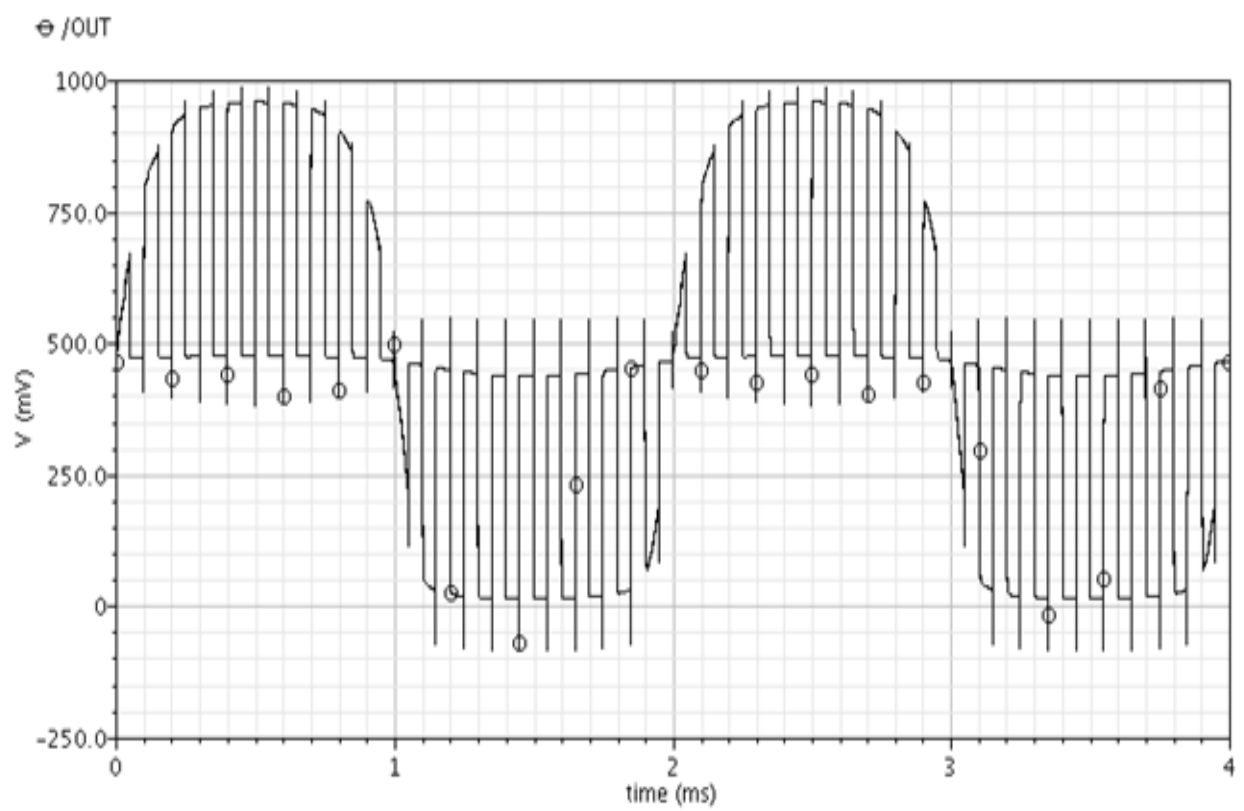

Figure 17. Output of Operational Amplifier without Low Pass Filter

Figure 18 indicates the simulation of the input referred noise spectrum with chopper or without chopper. Compared with non-chopper amplifier with chopper amplifier at $10 \mathrm{~Hz}$, the input referred noise of chopper amplifier is improved significantly, and when we using the chopper with dummy switch the input referred noise is much better( $13.5 \mathrm{nV} / \sqrt{\mathrm{Hz}})$.

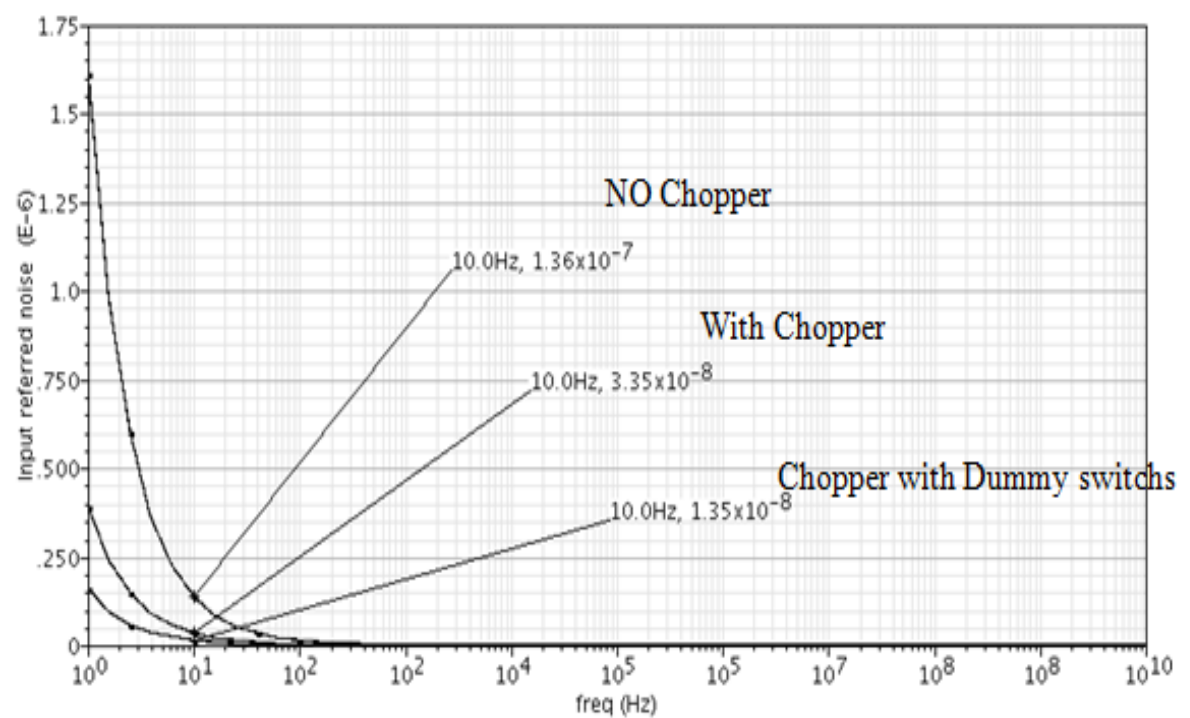

Figure 18. Balanced Two-stage Operational Amplifier Frequency Characteristic

Next the length of the transistor was fixed at the constant value of $0.1 \mathrm{um}$ and width was made to vary from $10 \mathrm{u}$ up to $50 \mathrm{um}$. According to the recorded results, the width variation 
resulted in almost a linear response with the total input referred noise figure magnitude, as shown in figure 19. The wider the transistor used the lower the total input referred noise contribution. Hence, it is important to set the width of the transistors in the modulator demodulator circuit by taking in to consideration the current level.

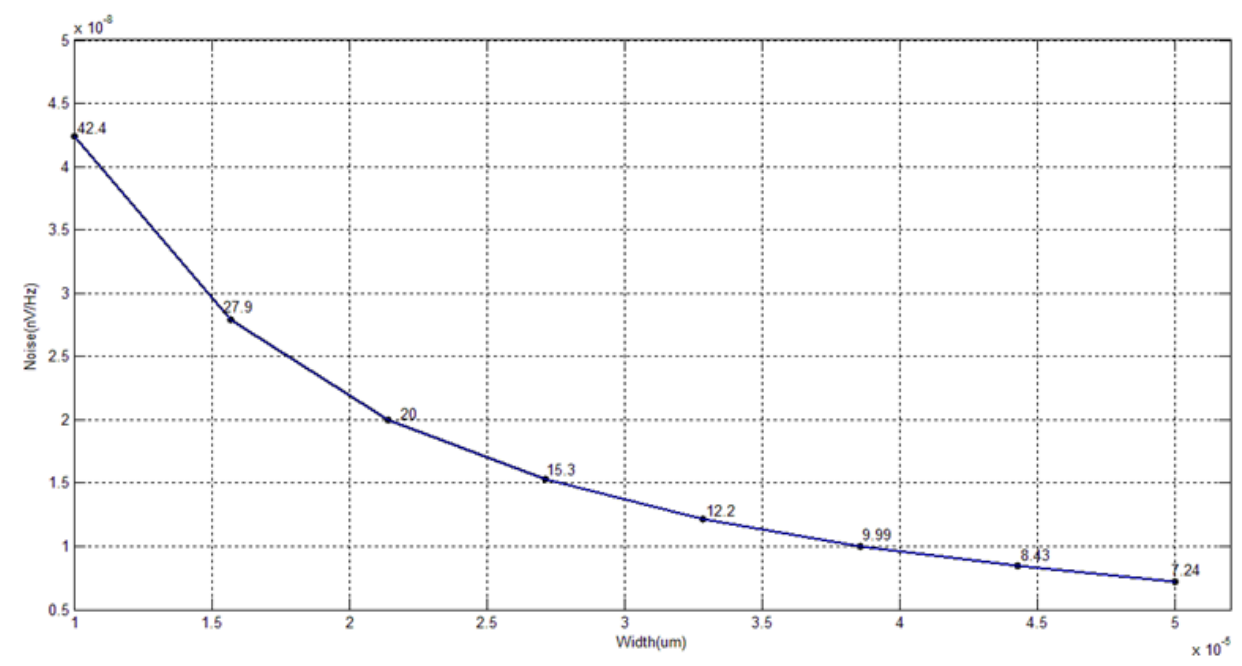

Figure 19. Effect of Transistor Width on Total Input Referred Noise

This a standard two-stage 11-transistor amplifier Figure 3 with miller compensation and zero-nulling active resistor $\mathrm{Rz}=(\mathrm{Rn} / / \mathrm{Rp})$. All the devices operate in saturation region except the transistors M9 and M10 operate in the linear region. The input differential stage draws $1 \mathrm{uA}$, while the output stage current is $1.1 \mathrm{uA}$. This results in a total current consumption of $2.1 \mathrm{uA}$ plus the current reference Iref $=0.5 \mathrm{uA}$, i.e. a $2.6 \mathrm{uW}$ power consumption from single 1V supply.

The overall performance summary of the complete circuit two-stage operational amplifier for biomedical applications is given in Table 3.

Table 3. Simulation Results of Schematic Two-stage Amplifier

\begin{tabular}{|c|c|}
\hline Process & 90nm CMOS \\
\hline Power supply & $1 \mathrm{~V}$ \\
\hline Power dissipation & $2.6 \mathrm{uW}$ \\
\hline CMRR & $131 \mathrm{~dB}$ \\
\hline Bandwidth & $15.3 \mathrm{kHz}$ \\
\hline PSRR+ & $70.7 \mathrm{~dB}$ \\
\hline Ad & $55.1 \mathrm{~dB}$ \\
\hline Input offset voltage (Vos) & $6.42 \mathrm{um}$ \\
\hline PM & 69.46 \\
\hline Input-referred-noise at $\mathbf{1 0 ~ H z}$ & $13.5 \mathrm{nV} / \sqrt{\mathrm{Hz}}$ \\
\hline
\end{tabular}

Table 4 shows a performance comparison between the proposed and currently used [13], [26], [29]. It can be seen that this work achieves the low input referred noise and lowest Power consumption. 
Table 4. Performance Comparison between Others Amplifier Circuits

\begin{tabular}{|l|c|c|c|c|}
\hline & This work & Ref.[13] & Ref.[26] & Ref.[29] \\
\hline Power supply(V) & 1 & 1.8 & 3.3 & 3.3 \\
\hline Technology CMOS (m) & $90 \mathrm{n}$ & $0.35 \mathrm{u}$ & $0.18 \mathrm{u}$ & $0.35 \mathrm{u}$ \\
\hline CMRR(dB) & 131 & 120 & 110 & - \\
\hline Amplifier voltage gain (dB) & 48 & 51 & - & 124.57 \\
\hline Power consumption (u W) & 2.6 & 775 & 420 & - \\
\hline Input offset voltage(uV) & 6.24 & - & 0.582 & $<60$ \\
\hline Input referred noise $(\mathrm{nV} / \sqrt{\mathrm{Hz}})$ @ $\mathbf{1 0 H z}$ & 13.5 & 56 & 22 & 312.86 \\
\hline
\end{tabular}

\section{Conclusion}

In this paper, we present a Two-stage operational amplifier topology for low voltage and low power, ECG Monitoring System applications, This two-stage amplifier with Miller compensation can be used in low power, low voltage High CMRR and PSRR applications such Biomedical instrument and a small battery operated devices. The circuit has been designed in a SPECTRE using 90nm CMOS technology.

To reduce the noise of the amplifier, we used the P-channel input devices with $\mathrm{N}$-channel load, because it's flicker noise is less than that the N-channel input devices with P-channel load.

We have described an ECG amplifier with low input-referred noise, 131-dB CMRR, less than $3 \mathrm{uW}$ of power consumption, and good cardiac signal fidelity.

Proposed two-stage operational amplifier with Miller compensation $(\mathrm{Cc})$ and nulling active resistor (Rz), is well suited to biomedical systems such as cardiac pacemaker, electrocardiogram (ECG) where low-power consumption is of primary concern.

using the chopper technique improve the input-referred-noise, even if we used the differential NMOS with dummy switches, there is a problem of spikes causing by clock feedthrough and charge injection, we can reducing these spikes by using another type of switch like , CMOS Transmission gate with dummy switches or we use other techniques.

\section{References}

[1] T. H. Ning, P. W. Cook, R. H. Dennard, C. M. Osburn, S. E. Schuster, and H. N. Yu, "1um MOSFET VLSI technology, Part IV-Hot-electron design constraints”, IEEE Trans.Electron.Devices, vol. 26,(1979), pp. 346353.

[2] M. F. Li, Uday Dasgupta, X. W. Zhang, and Yong Ching Lim, "A low-Voltage CMOS OTA with Rail-toRail Differential Input Range", IEEE Transactions on circuits and systems, vol. 47, (2000) January, pp.1-8.

[3] M. Swan, K. Arabi and B. Provost, "Implantable volume monitor and miniaturized simulator dedicated to bladder control”, Artificial Organs, vol. 21, no. 3, (1997), pp. 219-222.

[4] R. Stein, B. Charles, D. Davis, L. Jhamandas, J. A. Mannard, And T. R. Nichols, "Principles underlying new methods for chronic neural recording", Can. J. Of Neuro.SCI., (1975) August, pp. 235-244.

[5] D. Popovic, B. Stein, R. B. Jovanovic, K. L. Dai, A. R. Kostovand, and W.W. Armstrong, "Sensory nerve recording for closed-loop control to restore motor function", IEEE Trans. Biomed.Eng., vol. 40, no. 10, (1993) October, pp. 1024-1031.

[6] Y. Hu and M. Sawan, "CMOS Front-end Amplifier Dedicated to Monitor Very low Amplitude Signal from Imlantable Sensors”, Kluwer Academic Publishers. Manufactured in the Netherlands, vol. 33, (2002), pp. 2941.

[7] W.-S. Wang, Z.-C. Wu, H.-T. Huang and C.-H. Luo, "Low Power Instrumental Amplifier for Portable ECG", IEEE Circuits and Systems International Conference, (2009) April, pp. 1-4.

[8] C.-T. Ma, P.-I. Mak, M.-I Vai, P.-u. Mak, S.-H. Pun, W. Feng and R. P. Martins, "A 90nm CMOS BioPotential Signal Readout Front-End with Improved Powerline Interference Rejection", IEEECircuits and Systems, (2009) 24-27 May, pp. 665-668. 
[9] C.-C. Huang, S.-H. Hung and J.-F. Chung, "Front-End Amplifier of low-Noise and Tunable B/W Gain for Portable Biomedical Signal Acquidition”, IEEE Circuits and Systems, (2008) May, pp. 2717 - 2720.

[10] D. Yates, E. Lopez-Morillo and R. G. Carvajal, "A Low-Voltage Low-Power Front-End for wearable EEG Systems", conference of the IEEE EMBS, (2007) August.

[11] L. Lentola, A. Mozzi, A. Neviani and A. Baschirotto, "A 1uA Front-End for Pacemaker Atrial Sensing Channels", IEEE, (2009) September.

[12] C. Bronskowski and D. Schroeder, "An ultra-low-noise CMOS Operational Amplifier with programmable Noise-Power Trade-off ", IEEE Solid-State Circuits Conference,(2006) September , pp. 368 - 371

[13] Y. Hu and M. Sawan, "CMOS front-end amplifier dedicated to monitor very low amplitude signal from implantable sensors.” Analog Int. Circ. Signal Proc, (2002) October, vol. 33, pp. 29-41,

[14] N. Ahmed and N. Hesham, "A low Power low noise Capacitively Coupled Chopper Instrumentation Amplifier in 134 nm CMOS for Portable Biopotential Acquisition Systems", IEEE, (2013).

[15] L. Zhang and L. He, "system design of o low noise, low offset instrumentation amplifier with chopper stabilization" IEEE, 7th International Conference, (2007) October 22-25, pp. 627-630. .

[16] J. G. Webster, "Medical instrumentation: Application and design", $3^{\text {rd }}$ ed, John Wiley \& sons Inc., (1998).

[17] H.-C. Chow and B.-S. Tang, "A high Performance Current-mode Instrumentation Amplifier for Biomedical applications", $2^{\text {nd }}$ International Conference on Signal Processing Systems, (2010) July, pp. V2-64 - V2-67.

[18] C. Ken and L. Xiaoying, "A Zigbee Based Mesh Network for ECG Monitoring System”, Bioinformatics and Biomedical Engineering (iCBBE), (2010) June, pp. 1-4.

[19] Y. F. Low, I. B. Mustaffa, N. B. Mohd Saad and A. H. Bin Hamidon, "Development of PC-Based ECG Monitoring System", IEEE Research and Development, 4th Student Conference, (2006) June, pp. 66-69.

[20] PhysioBank, physiologic signal archives for biomedical research [Online]. Available: http://www.physionet.org/physiobank/

[21] The MIT-BIH Arrhythmia Database [Online]. Available: http:// physionet.org/physiobank/database/mitdb/

[22] The MIT-BIH Compression Test Database [Online]. Available: http:// www.physionet.org/physiobank/database/cdb/

[23] R. L. Geiger, P. E. Allen and N. R. Strader, "VLSI Design Techniques for Analog and Digital Circuits", McGraw-Hill, (1990).

[24] P. E. Allen and D. R Holberg, "CMOS Analog Circuit Design”, Second Edition, Oxford University Press, (2002).

[25] R. Wo, "Precision Instrumentation amplifiers and read-out IC for sensor Interfacing", Delft, 12 December (2012).

[26] A. Voulkidou and S. Siskos, "A Low Noise Low Offset Current Mode Instrumentation Amplifier", MIXDES 2012, 19th International Conference "Mixed Design of Integrated Circuits and Systems", (2012), May 24-26, Warsaw, Poland.

[27] N. Y. Sutri and J. O. Dennis, "Low-Noise, Low-offset Modulator Demodulator Circuit for Chopper Stabilization Technique in CMOS-MEMS Sensor Applications", IEEE, (2014), June, pp. 1-5.

[28] J. Nebhen, S. Meillère and M. Masmoudi, "A $250 \mu \mathrm{W} 0.194$ nV/rtHz Chopper-Stabilized Instrumentation Amplifier for MEMS Gas Sensor”, IEEE, International Conference on Design \& Technology of Integrated Systems in Nanoscale Era, (2012), 16-18 May, pp. 1-5.

[29] Q. Fu and X. Yan, “Analysis and Design od Instrumentation amplifier Based On Chopper Technology”, IEEE Laser Physics and Laser Technologies (RCSLPLT), (2010) July 28, pp. 318 - 321.

[30] E. J. Kennedy, "Operational Amplifier Circuits, Theory and Applications", Holt, Rinehart and Winston, Inc, New York, (1988). 
International Journal of Hybrid Information Technology Vol. 7, No. 6 (2014) 